\title{
Communication from the Editor-in-Chief: State of the ACM Transactions on Knowledge Discovery from Data
}

It was a pleasure serving as Co-Editor-in-Chief of the ACM Transactions on Knowledge Discovery from Data (ACM TKDD) with Xindong Wu from 2017 to 2020. Starting in December 2020, I have taken on full Editor-in-Chief responsibilities for the next three-year term. On behalf of ACM TKDD, and the editorial board in particular, I would like to thank Xindong for his dedicated service over the past three years. In this editorial, I hope to summarize some key recent developments and future plans for the journal.

The editorial board has greatly changed over the last year with a number of associate editors leaving and others joining in their place. Shuiwang Ji, Latifur Khan, Lingfei Wu, Suhang Wang, Karthik Subbian, and Paola Velardi have all joined as associate editors, bringing diverse skills to deal with the increasing influx of $\mathrm{p}$ apers from different ar eas. It ha s be come ne cessary to expand the editorial board over time as members retire or step down and the number of submitted manuscripts increases. The new post of Senior Associate Editor has been created to support me in managing this increase, and Xiangnan Kong has taken over this position. Several associate editors, including Bing Liu, Aristides Gionis, and Yizhou Sun, have recently left the board, and we thank them from their years of service to ACM TKDD.

One of our key goals in recent years has been to increase the timeliness of our review process. Authors work hard on submissions and spend months-in some cases years-producing manuscripts for the journal. The least we can do is treat their work with respect by providing timely reviews. The popularity of a journal to potential authors also relies on a high level of timeliness; they will be unwilling to submit manuscripts if it takes too long for ACM TKDD to reach a decision. As such, we have asked associate editors to avoid multiple major revision decisions so that manuscripts ideally get just one. We have also reduced the time allowed for major revisions to two months and for minor changes to one. The logic for this was that giving more time to authors does not guarantee a revision of higher quality. It can sometimes simply mean that revisions are made on a delayed schedule. If the paper is ultimately not accepted, too long a window will render the work too outdated for it to be revised for another venue. Asking the author to submit revisions earlier is good for the journal in terms of the currentness of its papers and for the author in terms of reducing the danger that a manuscript will become outdated.

ACM TKDD has seen a huge increase in submissions in recent years as a direct result of growing interest in topics related to deep learning. Although ACM TKDD was originally created to serve the data mining community, it has inevitably seen a rise in papers from those in machine learning. This trend has picked up in recent years because machine learning conferences have themselves expanded, in relation to data mining, and also because there is no ACM Transactions dedicated to

\footnotetext{
ACM Reference format:

Charu C. Aggarwal. 2021. Communication from the Editor-in-Chief: State of the ACM Transactions on Knowledge Discovery from Data. ACM Trans. Knowl. Discov. Data 16, 2, Article 21e (July 2021), 2 pages.

https://doi.org/10.1145/3463950
}

(C) 2021 Association for Computing Machinery.

1556-4681/2021/07-ART21e \$15.00

https://doi.org/10.1145/3463950 
machine learning. The below table demonstrates the clear increase in manuscripts submitted to TKDD.

\begin{tabular}{|l|c|c|c|c|}
\hline Year & $\mathbf{2 0 1 7}$ & $\mathbf{2 0 1 8}$ & $\mathbf{2 0 1 9}$ & $\mathbf{2 0 2 0}$ \\
\hline Manuscripts Submitted & 161 & 387 & 431 & 550 \\
\hline Manuscripts Accepted & 20 & 47 & 81 & 122 \\
\hline Manuscripts Published & 52 & 73 & 65 & 78 \\
\hline Pages Published & 1498 & 2134 & 1718 & 2095 \\
\hline Issues Published & 4 & 6 & 6 & 6 \\
\hline
\end{tabular}

The number of submissions rose from 160 in 2017 to 550 in 2020 at a more than three-fold increase! The number of manuscripts subsequently accepted has also gone up significantly, from 20 in 2017 to 122 in 2020, representing a six-fold increase. The acceptance rate has itself escalated, largely due to the higher quality of manuscripts flowing into the journal rather than any explicit instruction to the editorial board to accept more. We have greatly expanded the number of pages published to be able to accommodate the increased number of manuscripts.

Given the increased number of machine-learning papers being published at a journal originally dedicated to data mining, a question naturally arises about expanding the original intentions of ACM TKDD. This is a difficult question to answer as any change to agenda or title-even a minor one-risks re-indexing the journal as entirely new in libraries and databases. The decision is therefore a serious one, and we are in touch with ACM regarding how to expand our agenda without disrupting the journal's track record. Overall, I am thrilled at the huge increase in submissions that the journal has received over the last three years and believe that the future of ACM TKDD looks very bright indeed.

Charu C. Aggarwal Editor-in-Chief 